\title{
Editorial
}

\section{Biomarkers in respiratory diseases}

The December issue of Breathe focuses on biomarkers in respiratory diseases [1-4]. Biomarkers are measurable indicators of the presence, severity or type of a disease. They can help us understand the cause, phenotype, progression or regression, prognosis, or outcome of treatment of a disease. Biomarkers hold the promise of personalised medicine, which aims to tailor treatments to individual patients based on their biomarker profile and, by doing so, reduce the harms from ineffective treatments and increase the benefits from effective treatments. Biomarkers to describe disease phenotypes and to determine optimal treatments based on these phenotypes are receiving substantial attention in the current respiratory research literature. The search for clinically useful biomarkers that impact clinical decision-making is, however, challenging, and the vast majority of biomarkers are failing at the initial verification and validation stages before they enter clinical practice [5].

As clinicians we are sometimes left to wonder, after reading a research article or listening to a talk of a prominent researcher, how we can apply the latest evidence on biomarkers into our clinical practice. This issue of Breathe aims to provide clinicians with a summary and update on some of these biomarkers. Unfortunately, while there are clinical applications for biomarkers, many uncertainties remain, as highlighted in a review article on the clinical utility of fractional exhaled nitric oxide in the management of asthma and COPD [1] and a review on basing COPD treatment choices, namely (inhaled) corticosteroid treatment, on blood eosinophil levels [2]. Antimicrobial stewardship considerations have driven research on biomarker guidance to identify patients who might not require treatment with antibiotics. Meta-analyses of randomised controlled trials suggest that procalcitonin-guided antibiotic therapy in lower respiratory tract infections and exacerbation of COPD can reduce the use of antibiotics without compromising clinical outcomes [6, 7]. Nevertheless, despite this evidence, a review article in this issue [3] found that major guidelines generally do not recommend that decisions about the need for antibiotic therapy in respiratory diseases are based on procalcitonin levels, indicating that some important uncertainties remain. The review authors conclude that procalcitonin levels provide supportive information that can inform the decision about antibiotic treatment in respiratory diseases in conjunction with a thorough clinical assessment [3].

The Patient Voice article provides a rare insight into how a patient experiences the role of biomarkers in the management of her asthma [4]. From reading this article, you will appreciate how important it is to communicate to patients if and how biomarkers impact on their recommended treatment.

As another year draws to a close, I would like to thank all contributors, editorial board members, the hard-working team at the European Respiratory Society publications office and our readers. Happy holidays!

@ERSpublications

The December issue of Breathe focuses on biomarkers in respiratory diseases: read the introductory editorial by Chief Editor @ClaudiaCDobler http://bit.ly/36nzAiW

Cite as: Dobler CC. Biomarkers in respiratory diseases. Breathe 2019; 15: 265-266.

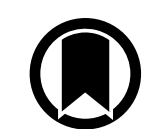

CrossMark 


\section{Affiliations}

\section{Claudia C. Dobler ${ }^{1,2}$}

${ }^{1}$ Institute for Evidence-Based Healthcare, Bond University, Robina, Australia. ${ }^{2}$ Dept of Respiratory Medicine, Liverpool Hospital, Sydney, Australia.

\section{Conflict of interest}

C.C. Dobler has nothing to disclose.

\section{References}

1. Turner SW, Chang AB, Yang IA. Clinical utility of exhaled nitric oxide fraction in the management of asthma and COPD. Breathe 2019; 15: 306-316.

2. Oliver B, Tonga K, Darley D, et al. COPD treatment choices based on blood eosinophils: are we there yet? Breathe 2019; 15: 318-323.

3. Creamer AW, Kent AE, Albur M. Procalcitonin in respiratory disease: use as a biomarker for diagnosis and guiding antibiotic therapy. Breathe 2019; 15: 296-304

4. Hamerlijnck D. Biomarker development in asthma from a patient's perspective. Breathe 2019; 15: 277-278.
5. Kern SE. Why your new cancer biomarker may never work: recurrent patterns and remarkable diversity in biomarker failures. Cancer Res 2012; 72: 6097-6101.

6. Schuetz P, Wirz Y, Sager R, et al. Effect of procalcitoninguided antibiotic treatment on mortality in acute respiratory infections: a patient level meta-analysis. Lancet Infect Dis 2018; 18: 95-107.

7. Mathioudakis AG, Chatzimavridou-Grigoriadou V, Corlateanu A, et al. Procalcitonin to guide antibiotic administration in COPD exacerbations: a meta-analysis. Eur Respir Rev 2017; 26: 160073. 